\title{
A!
}

This is an electronic reprint of the original article.

This reprint may differ from the original in pagination and typographic detail.

UI Haq, Faizan; Englund, Mikko; Antonov, Yury; Stadius, Kari; Kosunen, Marko; Östman, Kim; Koli, Kimmo; Ryynänen, Jussi

\section{A Blocker-Tolerant Two-Stage Harmonic-Rejection RF Front-End}

Published in:

IEEE Radio Frequency Integrated Circuits Symposium

DOI:

10.1109/RFIC.2019.8701765

Published: 01/01/2019

Document Version

Peer reviewed version

Please cite the original version:

Ul Haq, F., Englund, M., Antonov, Y., Stadius, K., Kosunen, M., Östman, K., Koli, K., \& Ryynänen, J. (2019). A Blocker-Tolerant Two-Stage Harmonic-Rejection RF Front-End. In IEEE Radio Frequency Integrated Circuits Symposium (pp. 203-206). (IEEE Radio Frequency Integrated Circuits Symposium digest of papers). IEEE. https://doi.org/10.1109/RFIC.2019.8701765

This material is protected by copyright and other intellectual property rights, and duplication or sale of all or part of any of the repository collections is not permitted, except that material may be duplicated by you for your research use or educational purposes in electronic or print form. You must obtain permission for any other use. Electronic or print copies may not be offered, whether for sale or otherwise to anyone who is not an authorised user. 


\title{
A Blocker-Tolerant Two-Stage Harmonic-Rejection RF Front-End
}

\author{
Faizan Ul Haq", Mikko Englund*, Yury Antonov\#, Kari Stadius", Marko Kosunen\#, Kim B. Östman ${ }^{\$}$, \\ Kimmo Koli*, Jussi Ryynänen ${ }^{\#}$ \\ \#Dept. of Electronics and Nanoengineering, Aalto University, Finland \\ \$Nordic Semiconductor Finland \\ *Huawei Technologies Oy Finland Co. Ltd. \\ ${ }^{1}$ faizan.ulhaq@aalto.fi
}

\begin{abstract}
SAW-less wideband receivers need to operate linearly in the presence of strong out-of-band blockers. In this paper, we introduce a blocker tolerant harmonic rejection RF front-end which is able to suppress blockers present at the local oscillator harmonics. The suppression is achieved by applying harmonic rejection in two stages, such that the first harmonic rejection already occurs at the output of LNA. The proposed front-end achieves this harmonic rejection with simpler 6-phase LO clocking and reduced number of base-band signal paths compared to 8-phase HR architectures. Further, the proposed design does not require any precise gain coefficients and implementing the harmonic rejection in two stages makes it more mismatch tolerant. In addition, near-band blocker linearity is improved by implementing a third order base-band feedback response which acts in conjunction with $\mathrm{N}$-path filtering. Implemented in a $28 \mathrm{~nm}$ FDSOI process, the front-end demonstrate 18-37dB harmonic rejection from the first stage and around 46-53dB of harmonic rejection from the second stage with a state-of-the-art blocker compression point of $2.5 \mathrm{dBm}$ for a third harmonic blocker and a near-band blocker compression point of $-6.5 \mathrm{dBm}$.
\end{abstract}

Keywords - Harmonic rejection, blocker tolerance.

\section{INTRODUCTION}

The emergence of multiple radio access standards have arisen the demand for wideband receivers which should be able to operate on multiple frequency bands. Such a receiver will need to perform in presence of strong out-of-band (OB) blockers which potentially saturate the receiver. Traditionally, these OB blockers have been attenuated through off-chip surface acoustic wave (SAW) filters which are not suitable for wideband receiver scenario due to their non-tunability. What is needed is an on-chip tunable filtering alternative for blocker suppression.

One such widely implemented on-chip filtering technique is N-path filtering, which offers moderate $\mathrm{Q}$ factor with a wide tuning range [1]. However, one of the shortcomings of original N-path filtering concept is its inability to suppress blockers at harmonics of the local-oscillator (LO) frequency. Various works [2], [3], [4], [5] have proposed harmonic rejecting N-path filtering. However, in order to achieve higher harmonic rejection $(\mathrm{HR})$, these techniques implement a higher number of paths together with the requirement of precise gain coefficients. Higher number of paths, inevitably lead to a complicated design with increased area and power consumption, while precise gain coefficients suffer from implementation inaccuracies. In addition, $\mathrm{HR}$ is usually implemented later in the receiver chain when gain has already been applied [3], [6]. Blocker at LO harmonics will thus already saturate the first stages before the HR is even takes place.

Some recent works [4], [5] have proposed HR at the output of first gain stage to improve receiver linearity for blockers at LO harmonics. Though these techniques achieve promising linearity improvements for blockers at LO harmonics, they still implement a higher number of $\mathrm{N}$-paths with the requirement of implementing precise gain coefficients.

In this paper, we propose a two-stage harmonic rejection RF front-end which achieves HR at the output of the first gain stage. This helps to improve front-end linearity for blockers at LO harmonics. The proposed architecture is simple as it utilizes a six-phase LO clock, compared with other implemented works which use eight phases or more [4], [5]. In addition, the proposed architecture does not require any precisely tuned gain coefficients while at the same time implementing the $\mathrm{HR}$ in two stages makes it more mismatch tolerant. We also propose a third-order base-band (BB) response together with an N-path filtering, which helps to greatly suppress near-band blockers.

The organization of this paper is as follows: Section II details the proposed two stage HR front-end while Section III elaborate the circuit design and measured results. Section IV ends in conclusion.

\section{Proposed Architecture}

The proposed two-stage harmonic-rejection front-end is presented in Fig. 1. The architecture implements three base-band downconversion paths each of which is $60^{\circ}$ degrees phase shifted from the adjacent path. This decreases the number of $\mathrm{BB}$ paths by one as compared to 8-phase $\mathrm{N}$-path downconversion [4], [5], [2]. From the three $60^{\circ}$ degree phase shifted BB outputs, conventional I and Q outputs can easily be derived in the digital domain after an ADC.

The first stage of $\mathrm{HR}$ rejection is implemented by two parallel low-noise amplifiers (LNA) together with six differential passive mixers clocked by six non-overlapping LO phases. The need for two LNA's and six mixers arise to create HR at the LNA output without using overlapping LO clocks which would otherwise decrease the LNA gain. The LO clocking for the passive mixers is presented in Fig. 2 which can be altered to either the 6-phase HR LO clock (blue), 


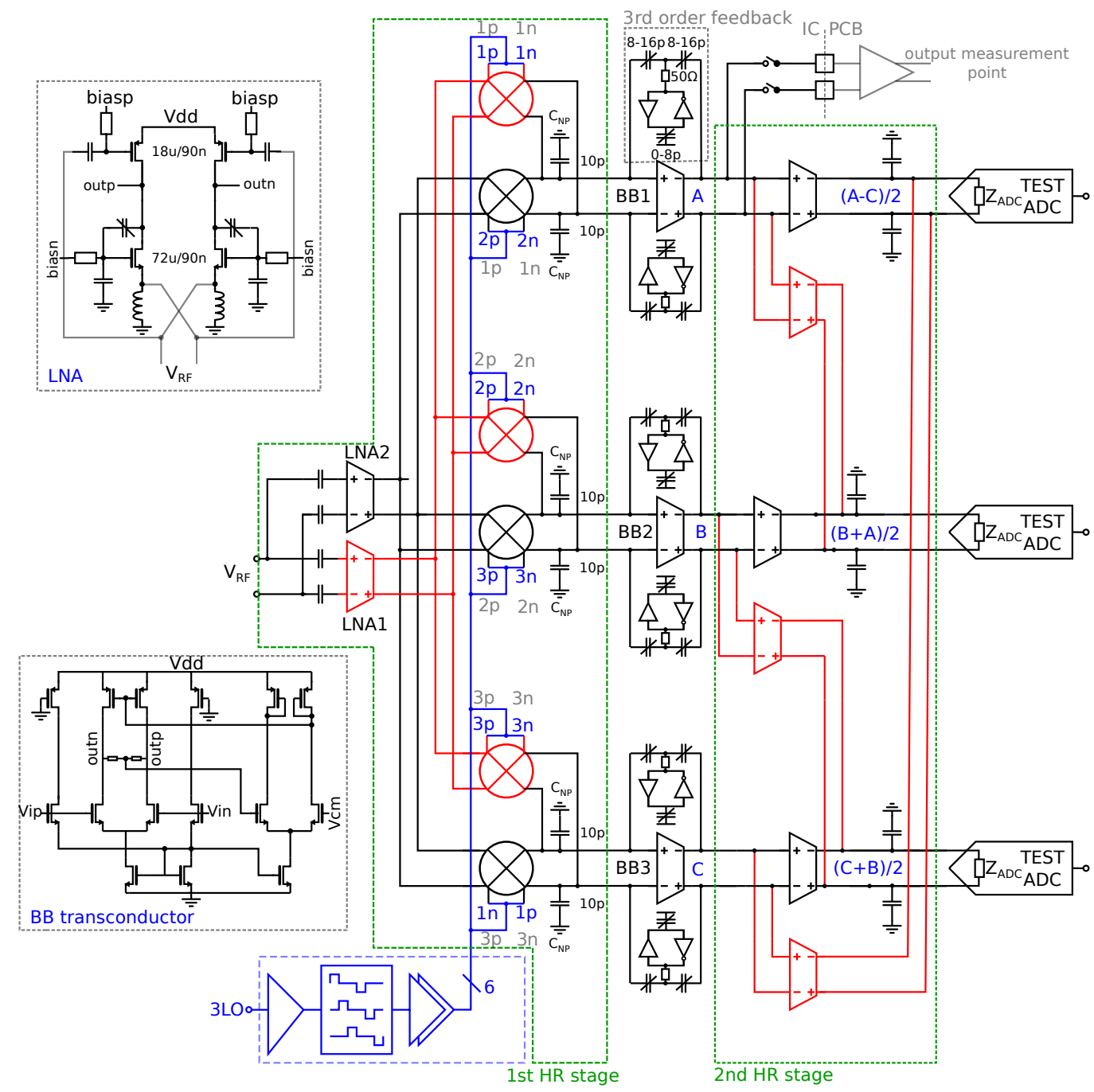

Fig. 1. Block diagram of proposed two stage harmonic rejection front-end.

or conventional 6-phase clock for lower noise contribution front-end (grey). Conventional LO clock is identical to having one equivalent LNA and three mixers while in the HR clocking arrangement, the functionality can be explained as follows: During LO phases $1 \mathrm{p}$ and $2 \mathrm{p}$, LNA1 and LNA2 get alternatively connected to BB1. In phase $3 p$ and $3 n$ neither LNA1 nor LNA2 are connected while in phases $1 \mathrm{n}$ and 2n, LNA1 and LNA2 get connected to BB1 with opposite polarity creating an effective LO at the input of the BB1 as shown in Fig. 2. Fourier analysis of HR LO waveform reveals ideally no third harmonic and therefore, creates third harmonic rejection with lower number of clock phases. Further, as the HR occurs exactly where the gain is applied, i.e. at the LNA output, front-end linearity in presence of blockers at the 3rd LO harmonic also improves. However, complete elimination of third harmonic is not possible due to device mismatches and LO non-idealities. Therefore, to improve the HR further second HR stage is required.

The second stage makes the effective downconverted signal more closely resemble a sine wave. To do this, we implement cross-coupled BB stages between three BB paths as shown in Fig. 1. This produces signals $(\mathrm{A}-\mathrm{C}) / 2,(\mathrm{~B}+\mathrm{A}) / 2$ and $(\mathrm{C}+\mathrm{B}) / 2$, where $\mathrm{A}, \mathrm{B}$ and $\mathrm{C}$ are the signal outputs from the first $\mathrm{BB}$ stages of three parallel $\mathrm{BB}$ paths. The generated signals $(\mathrm{A}-\mathrm{C}) / 2,(\mathrm{~B}+\mathrm{A}) / 2$ and $(\mathrm{C}+\mathrm{B}) / 2$ resemble a sine wave more closely and simple Fourier analysis shows increased harmonic rejection.

In order to improve the near-band blocker attenuation we propose a third-order BB filtering stage as shown in Fig. 1. The response is implemented with a third-order feedback impedance, where the equivalent inductance has been implemented with gyrator transconductors for on-chip fabrication. The created response works in conjunction with $\mathrm{N}$-path filtering to attain a sharp third-order band-pass response at LNA output, consequently, improving the near-band blocker linearity.

\section{CiRCUIT DESIGN AND MEASUREMENTS}

The proposed front-end was fabricated in a $28 \mathrm{~nm}$ FDSOI technology for an operating frequency of $0.7-2 \mathrm{GHz}$. The front-end was part of complete receiver, with $\Delta \Sigma$ ADC and digital processing. The chip photograph of complete receiver 


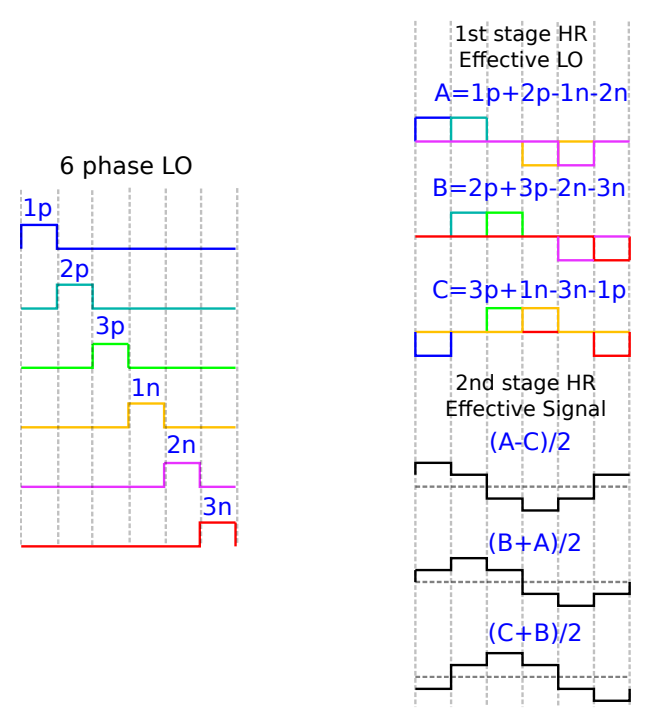

Fig. 2. Conceptual representation of proposed two stage HR with the generated effective LO at each HR stage.

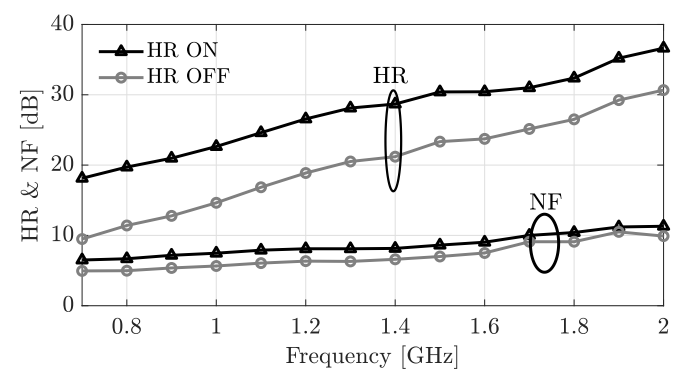

Fig. 3. NF and the first stage HR of the proposed front-end, for HR LO clocking (black) and conventional LO clocking (grey).

IC is presented in Fig. 7, in which the front-end part together with LO generator occupies an active area of $0.65 \mathrm{~mm}^{2}$. In order to measure the performance of the first HR stage, a test signal from the output of the first $\mathrm{BB}$ amplifier was routed outside the IC. RF performance was then measured by buffering this signal with an external operational amplifier and observing response at output measurement point as shown in Fig. 1.

On the circuit level, the implemented LNA's consist of push-pull common-gate common-source amplifiers with capacitive feedback for impedance matching, while the passive mixers were implemented with large aspect ratio transistors to ensure small switch resistance. The BB integrators were implemented with a dynamically-biased differential pair where the transconductance of the first $\mathrm{BB}$ stage was designed to be eight times higher than transconductance of later stage for lower noise contribution. The capacitors $\mathrm{C}_{\mathrm{NP}}$ provide additional blocker rejection at higher frequency offsets where the bandwidth of the first BB stage is not sufficient for a reasonable feedback response. For LO generation, an external signal at $3 \mathrm{f}_{\mathrm{LO}}$ was provided to $\mathrm{LO}$ chain which was then divided by three to create six non-overlapping LO clocks with $16.7 \%$ dutycycle, and finally routed to passive mixers through inverter buffers.

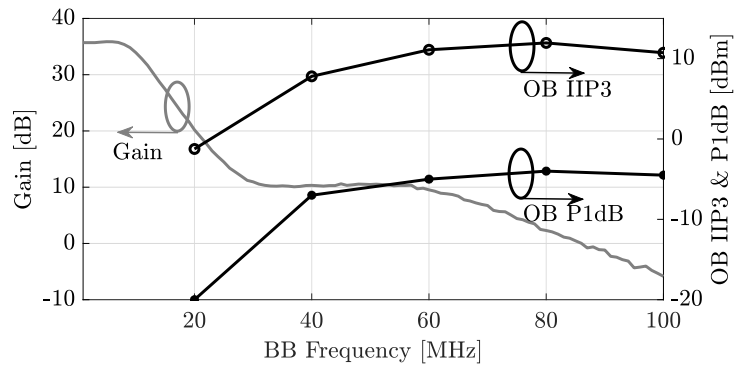

Fig. 4. $\mathrm{BB}$ filtering response, $\mathrm{OB}$ IIP 3 and $1 \mathrm{~dB}$ compression point vs. $\mathrm{BB}$ frequency for an $\mathrm{f}_{\mathrm{LO}}=1.5 \mathrm{GHz}$. Third order $\mathrm{BB}$ filtering response gets upconverted to LNA output nodes with $\mathrm{N}$-path filtering consequently helping to suppress near-band blockers.

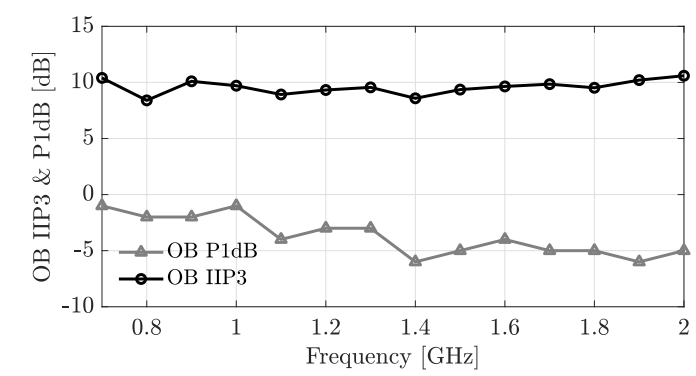

Fig. 5. OB IIP3 and P1dB versus front-end operating frequency. The blocker is placed at $\mathrm{f}_{\mathrm{LO}}+100 \mathrm{MHz}$

Fig. 3 shows the measured NF and HR of the first stage across the operating frequency range. HR in the range of 18 to $37 \mathrm{~dB}$ is observed for HR LO clock. One of the consequence of the HR LO clock is noise circulation from one base-band path to other. This happens because a single LNA is connected to two base-band paths at all time instances, resulting in creation of the noise circulation path and a higher $\mathrm{NF}$ front-end. To validate the noise circulation, we have also implemented an LO clocking mode where each BB branch is only connected to one LNA at a time, thus eliminating the noise circulation path. With this $\mathrm{LO}$ arrangement around $3 \mathrm{~dB}$ improvement in front-end NF is observed in measurements. The increase of NF at high frequencies was due to non-optimal layout design of LO chain which resulted in overlapping of LO signals at higher frequencies resulting in increased NF. A careful re-design of LO chain should reduce this increase of NF.

In Fig. 4, the third order BB filtering response, OB IIP3 and $\mathrm{P} 1 \mathrm{~dB}$ compression points are plotted versus BB frequency offset. One can observe third order filtering near the BB bandwidth of $10 \mathrm{MHz}$. This third order response is upconverted to LNA output nodes with N-path filtering, consequently, helping to suppress near-band blockers. For example, the measured $\mathrm{P} 1 \mathrm{~dB}$ compression point for a near-band blocker located at $40 \mathrm{MHz}$ offset from $\mathrm{f}_{\mathrm{LO}}$ is $-6.5 \mathrm{dBm}$ which is just $1.5 \mathrm{~dB}$ less than $\mathrm{P} 1 \mathrm{~dB}$ measured at a $100 \mathrm{MHz}$ offset. To observe the front-end linearity behavior across the operating frequency Fig. 5 shows the measured OB IIP3 and P1dB versus frequency for a blocker at $100 \mathrm{MHz}$ offset from LO. 
Table 1. Performance summary and comparison

\begin{tabular}{|c|c|c|c|c|c|}
\hline & This work & [4] & [5] & [6] & [7] \\
\hline Carrier Frequency $(\mathrm{GHz})$ & $0.7-2$ & $0.2-1$ & $0.1-2.4$ & $0.7-1.4$ & $0.5-3$ \\
\hline $\mathrm{HR}$ at first gain stage $(\mathrm{dB})$ & $18-37$ & 20 & $52-54$ & NA & 35 \\
\hline Second stage $\mathrm{HR}^{2}(\mathrm{~dB})$ & $46-53$ & $51-52$ & NA & $40-67$ & NA \\
\hline N-path phases & 6-phase & 8-phase & 8-phase & 8-phase & 8-phase \\
\hline Blocker input P1dB (dBm) & -1 to $-5 @ 10 f_{\mathrm{BW}}$ & $-2.4 @ 10 f_{B W}$ & -6 to $2.5 @ 27 f_{\mathrm{BW}}$ & -8.5 to $10 @ 10 f_{\mathrm{BW}}$ & - \\
\hline Blocker input P1dB@ @ $3 \mathrm{f}_{\mathrm{LO}}(\mathrm{dBm})$ & $2.5 @ 10 f_{\mathrm{BW}}$ & -2.8 & -8 & NR & NR \\
\hline Near band blocker P1dB $(\mathrm{dBm})$ & $-6.5 @ 4 f_{B W}$ & NR & NR & NR & -22 to $-4 @ 4 f_{(L O}$ \\
\hline Noise Figure $(\mathrm{dB})$ & $5-11$ & $5.4-6$ & 1.7 & $1.5-8$ & $3.8-4.7$ \\
\hline OB IIP3 $(\mathrm{dBm})$ & $8-11 @ 10 f_{B W}$ & $9 @ 10 f_{\mathrm{BW}}$ & 10 & $1-20.5 @ 10 f_{\mathrm{BW}}$ & -20 to $-4.8 @ 4 f_{\mathrm{BW}}$ \\
\hline Gain $(\mathrm{dB})$ & 36 & 36 & NR & $20.8-36.8$ & 50 \\
\hline $\mathrm{BB}$ bandwidth $\left(\mathrm{f}_{\mathrm{BW}}\right)(\mathrm{MHz})$ & 10 & 2 & $0.2-3$ & 10 & 10 \\
\hline Power (mW)@Supply voltage (V) & $54 @ 1$ & $26-32 @ 1.2,2.5$ & 36.8-62.4@1 & $52 @ 0.8,1,1.2$ & 76-168@NR ${ }^{(1}$ \\
\hline Process & 28nm FDSOI & $65 \mathrm{~nm}$ & $28 \mathrm{~nm}$ & $65 \mathrm{~nm}$ & $65 \mathrm{~nm}$ \\
\hline
\end{tabular}

NR: not reported, NA: not applicable. 1) signal path 2) 1st and 2nd stage combined HR.

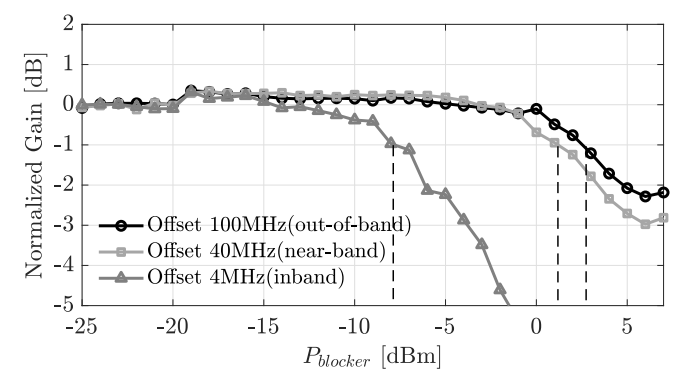

Fig. 6. Measured signal gain under the presence of strong blocker at $3 \mathrm{f}_{\mathrm{LO}}$. For a blocker at $3 \mathrm{f}_{\mathrm{LO}}+100 \mathrm{MHz}$, blocker P1dB of $2.5 \mathrm{dBm}$ is observed.

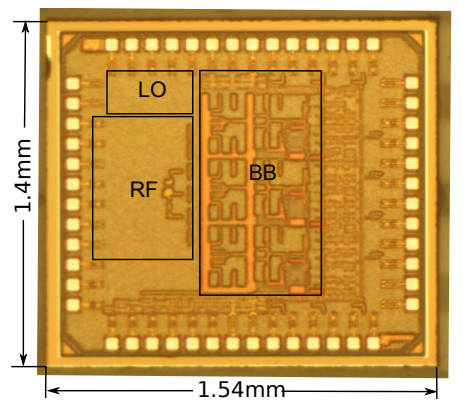

Fig. 7. Chip photograph of the proposed harmonic rejection receiver.

For observing front-end linearity in the presence of blockers at $3 \mathrm{f}_{\mathrm{LO}}$, linearity was measured with three offset frequencies, $100 \mathrm{MHz}, 40 \mathrm{MHz}$ and $4 \mathrm{MHz}$. These three offsets represents downconversion of the blocker to out-of-band, near-band, and in-band reception frequencies. The measurement was performed by sweeping the blocker power and determining the $\mathrm{P} 1 \mathrm{~dB}$ point of the receiver. We observe P1dB points of $-7,1$ and 2.5 respectively as shown in Fig. 6 achieving state-of-the-art results [4], [5].

\section{CONCLUSION}

Blocker tolerance is one of the key specifications for SAW-less wideband receivers. In this paper, we have proposed a blocker tolerant harmonic rejection front-end which is able to efficiently suppress blockers present at LO harmonics together with suppression of near-band blockers. This is achieved by implementing HR in two stages: Output of the LNA and in the second BB stage. Further, a third order BB impedance is implemented which acts in conjunction with N-path filtering for near-band blocker attenuation. The proposed architecture uses a lower number of LO phases and BB paths compared to 8-phase HR architectures and achieves HR without implementation of precise gain coefficients. Measured results from a fabricated prototype implemented in $28 \mathrm{~nm}$ FDSOI technology demonstrate $18-37 \mathrm{~dB}$ HR from the first stage and around 46-53dB of HR from second stage with a near-band blocker compression point of $-6.5 \mathrm{dBm}$ and an impressive $\mathrm{BCP}$ of $2.5 \mathrm{dBm}$ for third harmonic blocker.

\section{ACKNOWLEDGMENT}

This work has been supported by the Academy of Finland, and European Unions Horizon 2020 Research and Innovation Programme under the Marie Sklodowska-Curie Grant 704947.

\section{REFERENCES}

[1] A. Ghaffari, E. A. M. Klumperink, M. C. M. Soer, and B. Nauta, "Tunable high-Q N-path band-pass filters: Modeling and verification," IEEE Journal of Solid-State Circuits, vol. 46, no. 5, pp. 998-1010, May 2011.

[2] X. Zhang, B. Chi, and Z. Wang, "A 0.11.5 GHz harmonic rejection receiver front-end with phase ambiguity correction, vector gain calibration and blocker-resilient TIA," IEEE Transactions on Circuits and Systems I: Regular Papers, vol. 62, no. 4, pp. 1005-1014, April 2015.

[3] A. Nejdel, M. Törmänen, and H. Sjöland, "A $0.73 .7 \mathrm{GHz}$ six phase receiver front-end with third order harmonic rejection," in 2013 Proceedings of the ESSCIRC (ESSCIRC), Sep. 2013, pp. 279-282.

[4] Y. Xu, J. Zhu, and P. R. Kinget, "A blocker-tolerant RF front end with harmonic-rejecting N-path filter," IEEE Journal of Solid-State Circuits, vol. 53, no. 2, pp. 327-339, Feb 2018.

[5] D. Murphy, H. Darabi, and H. Xu, "A noise-cancelling receiver resilient to large harmonic blockers," IEEE Journal of Solid-State Circuits, vol. 50, no. 6, pp. 1336-1350, June 2015.

[6] J. Musayev and A. Liscidini, "Quantized analog RX front-end for SAW-less applications," in ESSCIRC 2018 - IEEE 44th European Solid State Circuits Conference (ESSCIRC), Sep. 2018, pp. 306-309.

[7] R. Chen and H. Hashemi, "Reconfigurable receiver with radio-frequency current-mode complex signal processing supporting carrier aggregation," IEEE Journal of Solid-State Circuits, vol. 50, no. 12, pp. 3032-3046, Dec 2015. 